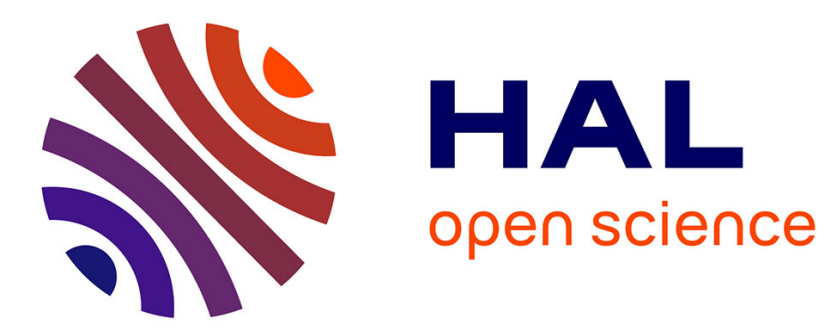

\title{
Le coppie bi-nazionali: norme europee e esperienze nazionali
}

Paola Panzeri, Gianluigi Moscato, Laura Odasso, Juan Antonio Domínguez

\section{To cite this version:}

Paola Panzeri, Gianluigi Moscato, Laura Odasso, Juan Antonio Domínguez. Le coppie bi-nazionali: norme europee e esperienze nazionali. Psicologia di comunità, 2013. hal-01686933

\section{HAL Id: hal-01686933 https://hal.science/hal-01686933}

Submitted on 17 Jan 2018

HAL is a multi-disciplinary open access archive for the deposit and dissemination of scientific research documents, whether they are published or not. The documents may come from teaching and research institutions in France or abroad, or from public or private research centers.
L'archive ouverte pluridisciplinaire HAL, est destinée au dépôt et à la diffusion de documents scientifiques de niveau recherche, publiés ou non, émanant des établissements d'enseignement et de recherche français ou étrangers, des laboratoires publics ou privés. 


\title{
Le coppie bi-nazionali: \\ norme europee e esperienze nazionali
}

\author{
di Paola Panzeri*, Gianluigi Moscato**, \\ Laura Odasso $^{* * *}$ e Juan Antonio Domínguez ${ }^{* * * *}$
}

\section{L'Unione Europea: un quadro degli strumenti normativi sulle unio- ni bi-nazionali}

In Europa, tra il 2008 e il 2010, un matrimonio su dodici è stato binazionale, cioè contratto tra individui di nazionalità differente. Tale dato, variabile secondo i paesi (uno su cinque in Svizzera e uno su mille in Romania) esclude le coppie conviventi more uxorio, cioè quelle non sposate (Eurostat, 2012); tuttavia si tratta di un indicatore che permette di evidenziare una realtà sociale cui, al momento, non corrisponde ancora una realtà politica.

Concentrando l'analisi sull'Unione Europea, con i suoi confini attuali e il suo corpus normativo, è necessario fare qualche considerazione preliminare. Innanzitutto, le politiche familiari sono principalmente di competenza degli Stati Membri e non dell'Unione ${ }^{1}$. Questo significa che la capacità di legiferare dell’Unione è limitata. Inoltre, è opportuno precisare la

* Analista di politiche europee, COFACE - Confederazione delle Associazioni Familiari dell'Unione Europea, e-mail: ppanzeri@coface-eu.org.

** Docente Università di Malaga e portavoce accademico di Asfamix, e-mail: moscato@uma.es.

${ }^{* * *}$ Dottore in Sociologia e in Storia e Istituzioni dell'area Mediterranea, Università Ca' Foscari di Venezia e Université de Strasbourg, membro de Les Amoureux au Ban Public, email: la.odasso@gmail.com.

${ }_{* * * *}$ Presidente Asfamix, e-mail: Presidente Asfamix, hogares@asfamix.es.

${ }^{1}$ La divisione delle competenze tra Stati Membri e Unione Europea è fissata dal trattato di Lisbona, che regola l'Unione e il suo funzionamento. Tale Trattato è concordato dagli Stati Membri e pertanto anche la decisione di quali competenze affidare in maniera esclusiva o parziale all'Unione è frutto di una negoziazione tra i Governi degli Stati Membri. Per conoscere meglio la ripartizione delle competenze si può consultare la pagina http://europa.eu/ legislation_summaries/institutional_affairs/treaties/lisbon_treaty/ai0020_it.htm

Psicologia di comunità, n. 1/2013 
terminologia e parlare di coppie bi-nazionali e non di coppie miste o interculturali, poiché è la differente nazionalità dei due membri della coppia che definisce l'applicabilità di tali norme (Odasso, 2013; Collet, 2012).

Basandosi sulle precedenti affermazioni è quindi necessario distinguere tre categorie di coppie bi-nazionali. La prima, quella in cui entrambi i membri della coppia sono cittadini di un paese dell'UE, la seconda quella in cui la coppia è composta da un cittadino europeo e da un cittadino di un paese terzo e la terza che è rappresentata dalle coppie in cui entrambi i membri sono cittadini di paesi terzi.

Queste distinzioni sono valide, in principio, sia per le coppie eterosessuali che omosessuali, per coppie sposate o con unioni civili ufficialmente registrate, a condizione che nel paese di residenza della coppia la loro unione sia riconosciuta ${ }^{1}$. Tuttavia, per le coppie conviventi o la cui unione non sia riconosciuta sussistono difficoltà ulteriori, in particolare se uno dei due partner è cittadino di un paese terzo (es.: difficoltà per ottenere/rinnovare il permesso di soggiorno, etc.) o se la coppia si muove o si trasferisce in un altro paese dell'UE (es.: riconoscimento dell'unione tra $\mathrm{i}$ partner, patria potestà per figli adottivi, etc.).

L'Unione Europea possiede degli strumenti legislativi che regolano l'accesso, la circolazione e la residenza nei suoi Stati membri. Per capire quindi come le norme europee influenzano la formazione delle famiglie binazionali, è necessario tornare alla classificazione descritta in precedenza.

Se la coppia è composta da due cittadini europei, entrambi godono del diritto di libera circolazione e le pratiche per la regolarizzazione della loro unione sono prettamente amministrative e dipendono in gran parte dallo Stato in cui la coppia decide di stabilire la residenza.

Nel caso in cui entrambi i partner sono cittadini di paesi terzi, molto dipende dal titolo di soggiorno in possesso da uno o da entrambi i partner nell'Unione Europea, se entrambi sono già presenti sul territorio dell'UE $\mathrm{o}$ se si necessita di un ricongiungimento familiare. Per quest'ultimo caso, è in vigore una direttiva $\left(2003 / 86 / \mathrm{CE}^{2}\right)$ che determina le modalità e le restrizioni a tale pratica. Attualmente, a seguito di consultazioni pubbliche alle quali la società civile si è espressa in maniera compatta sulla possibilità di rinegoziare il contenuto della direttiva, la Commissione Europea ha afferma-

\footnotetext{
${ }^{1} \mathrm{Al}$ momento della redazione, nove paesi dell'Unione Europea su ventisette hanno una legislazione che consenta le unioni tra coppie dello stesso sesso, e sedici paesi dell'Unione su ventisette riconoscono le unioni civili registrate. La legislazione in materia di unioni civili registrate varia molto da Stato a Stato. Per ulteriori informazioni consultare la pagina "Famiglia" del portale "La tua Europa" dell'Unione Europea: http://europa.eu/youreurope/citizens/

2 http://eurlex.europa.eu/LexUriServ/LexUriServ.do?uri = OJ:L:2003:251:0012:0018:it: pdf
} 
to di voler produrre delle linee guida che supportino gli Stati membri in una migliore attuazione della direttiva stessa ${ }^{3}$.

L'ultima tipologia che prenderemo in considerazione è la coppia formata da un cittadino europeo e da un cittadino non-europeo. In questi casi, la direttiva 2004/38/CE ${ }^{4}$ stabilisce il quadro normativo per la circolazione e il soggiorno di cittadini UE e i loro familiari. Tuttavia, alla luce delle differenze nelle normative nazionali sulla famiglia e delle diverse restrizioni che sono messe in atto nei diversi Stati europei, numerose sono le difficoltà incontrate dalle coppie bi-nazionali, anche se uno dei due partner è cittadino europeo. Le normative di alcuni Stati membri pongono delle restrizioni o delle difficoltà tali che, in vari casi si è assistito all'utilizzo della cosiddetta "strada Europea", cioè il trasferimento della coppia in un altro paese dell'Unione al fine di ricorrere alle norme europee - come la direttiva 2004/38/CE - meno restrittive.

Di fronte a queste problematiche, le risposte messe in campo a livello europeo e nazionale sono state molteplici. Tra le risposte europee innanzitutto la creazione, nel 1987, della figura del "Mediatore Europeo per i casi di sottrazione internazionale di minori" assistendo i membri delle coppie nella ricerca di una soluzione, e il cui principio guida è l'interesse superiore del minore 5 .

Inoltre, possiamo annoverare la proposta legislativa, attualmente in studio al Parlamento Europeo, per regolare i regimi di proprietà matrimoniale, o l'audizione pubblica al Parlamento Europeo sulla libera circolazione delle famiglie LGBT.

Infine, è utile citare la realizzazione, con il contributo della Commissione Europea, di un sito internet che raccoglie le normative dei paesi membri dell'Unione Europea sul tema delle famiglie bi-nazionali ${ }^{6}$.

${ }^{3}$ COFACE, Confederazione delle Associazioni Familiari dell'UE (www.coface-eu.org) si è da subito mobilitata con altre Organizzazioni non Governative per sottolineare le criticità della presente direttiva e per suggerire aree di miglioramento. Il 15 maggio 2012 un documento sottoscritto da 75 organizzazioni è stato inviato alla stampa e alla Commissione Europea. Il documento è disponibile alla pagina http://www.coface-eu.org/en/Press-Media/ Press-Releases/

${ }^{4}$ Il testo della direttiva 2004/38/CE è disponibile sul sito della Commissione Europea: http://eurlex.europa.eu/LexUriServ/LexUriServ.do?uri = OJ:L:2004:229:0035:0048:it:pdf http://ec.europa.eu/justice/citizen/files/guide_2004_38_ec_en.pdf

${ }^{5}$ Per ulteriori informazioni sulla figura, la funzione e per contattare il mediatore, è possibile visitare la pagina: http://www.europarl.europa.eu/aboutparliament/it/000c205a13/ Mediatore-europeo-per-i-casi-di-sottrazione-di-minori.html

${ }^{6} \mathrm{http}: / / \mathrm{www}$. coupleseurope.eu/it/home 


\section{Alcuni esempi nazionali: l'azione della società civile}

Se le normative rendono più difficoltosa la formazione di una coppia binazionale, la società civile si è organizzata per dare risposte concrete ai bisogni giuridico amministrativi vissuti da tali individui. In vari paesi, le famiglie bi-nazionali e miste si sono organizzate in associazioni locali o nazionali per dar voce alle loro istanze, offrire supporto a chi si trova in difficoltà e promuovere campagne di sensibilizzazione e lobbying al fine di proporre modifiche ad alcuni specifici aspetti della normativa nazionale. Negli ultimi anni, qualche passo avanti è stato compiuto e la dimensione europea si è affacciata nel campo di azione delle singole associazioni locali e nazionali. In questo senso, vogliamo segnalare i due congressi che la Coordinazione europea per il diritto degli stranieri di vivere in famiglia (Coordination Européenne pour le Droit des Etrangers à Vivre en Famille, Bruxelles, CoordEurop) e l'Unione Nazionale delle associazioni familiari spagnole (Union de Asociaciones Familiares, UNAF Madrid) ha organizzato sul tema ${ }^{7}$.

Nelle sezioni seguenti verrà presentata l'esperienza di tre associazioni (Les Amoureux au ban public, Francia; ASFAMIX, Spagna e Verband binationaler Familien und Partnerschaften e.V., Germania). Il percorso di ciascuna di esse mette in rilievo delle differenze nazionali, ma anche alcune somiglianze di approccio e di visione.

\subsection{L'esperienza francese: Les Amoureux au ban public}

L'esperienza de Les Amoureux au ban public ${ }^{8}$ nasce nel 2007 a Montpellier, per rispondere al crescente numero di coppie franco-straniere che si rivolgevano al servizio d'accoglienza giuridica della sede locale de $\mathrm{LaCi}$ made .

Nel 2003, infatti, la legge francese crea il reato di «matrimonio di inte-

${ }^{7}$ Per il seminario transnazionale 2010 tenuto a Murcia, Spagna, consultare la pagina dedicata: http://www.coordeurop.org/fr/134_Les_familles_interculturelles_un_defis_et_un chance. Per il convegno 2012, organizzato in collaborazione con l'Università di Malaga, (19-20 aprile 2012), si veda: http://www.unaf.org/jornadas_seminario_transnacional_malaga. html

${ }^{8}$ Il cui nome trae ispirazione dalla canzone Les Amoureux des bancs publics del cantante francese Georges Brassens (1954) e gioca sull'omofonia dei termini «bancs» (panchine) e «ban» (pubblicazione), in riferimento alle pubblicazioni di matrimonio.

${ }^{9}$ Creata nel 1939, La Cimade è un'associazione che si occupa dell'accompagnamento giuridico dei migranti, dei richiedenti asilo e dei rifugiati. Per ulteriori informazioni si veda il sito internet: http://www.cimade.org/ 
resse» e, negli anni successivi, chi vuole sposare un cittadino proveniente da un paese terzo deve intraprendere un vero e proprio percorso a ostacoli (Ferran, 2009). Gli interrogatori preventivi e le inchieste sono diventate una prassi ordinaria, anche per chi si sposa all'estero. In questo caso, la procedura prevede dei colloqui separati per i futuri coniugi presso il consolato francese e, dopo ulteriori verifiche, una volta trascritto il matrimonio in Francia, il coniuge straniero deve fare richiesta di ricongiungimento, quindi attendere un visto per raggiungere la Francia. Lo stesso visto di lungo soggiorno, istituito dalla legge del 2006, deve essere richiesto dal migrante irregolare che si sposa in suolo francese, in questo caso a seguito del matrimonio il coniuge straniero sprovvisto di permesso deve rientrare nel paese di origine e richiedere un visto i cui tempi di rilascio sono molto lunghi senza contare i rifiuti (La Cimade, 2010; Gisti et al., 2009).

Anche a seguito del matrimonio, la necessità di provare la coabitazione persiste. Il coniuge straniero di un(a) francese può fare richiesta della nazionalità francese dopo quattro anni di vita coniugale comune e ininterrotta (cinque se la residenza in Francia non è continuativa) dando prova di integrazione alla società francese e di un grado sufficiente di conoscenza linguistica; anche il termine per il rilascio della carta di residente è aumentato ${ }^{10}$.

L'emergenza di tali problematiche pressoché sconosciute all'opinione pubblica porta un gruppo di giuristi e attivisti de La Cimade di Montpellier a creare il progetto de Les Amoureux au Ban Public con lo scopo di difendere il diritto di tali coppie a condurre una vita familiare normale in un quadro legislativo e amministrativo sempre più ostico. In altre città, decine di coppie che incontrano difficoltà amministrative e persone sensibili al tema creano simili collettivi. L'obiettivo, infatti, degli ideatori del movimento è l'implicazione diretta delle coppie nelle azioni e nelle decisioni per raggiungere un grado di autonomia giuridica, una rete di mutuo aiuto e di coscienza delle possibilità di difesa dei propri diritti (Odasso, 2013).

Nel 2010 viene creata l'associazione di sostegno al movimento (Association de soutien aux Amoureux au ban public), che non ha tuttavia il potere di rappresentare il movimento, all'interno del quale i collettivi locali mantengono la loro autonomia d'azione, benché l'obiettivo sia comunque comune. Parallelamente, un coordinamento nazionale centralizza alcune pratiche, organizza alcune iniziative e è l'interlocutore della lobbying politico-amministrativa.

${ }^{10}$ Nel 2009 in Francia viene coniato un nuovo concetto il "matrimonio grigio" (mariage gris), si tratta di quel matrimonio, in cui il coniuge francese viene circuito dal coniuge straniero che vorrebbe sposarlo al solo fine di regolarizzare la sua situazione e/o di raggiungere la Francia, a differenza del matrimonio cosiddetto "bianco" (mariage blanc), in cui entrambi i coniugi sarebbero complici nell'atto di matrimonio di interesse. 
L'azione si articola su tre fronti: (1) l'accoglienza specializzata per assicurare la difesa delle coppie sul piano giuridico (nel 2012 i collettivi hanno seguito 1236 coppie); (2) l'organizzazione di azioni di sensibilizzazione e di informazione destinate all'opinione pubblica, ai media e agli attori del mondo associativo e sindacale (es.: petizioni, azioni urgenti, comunicati stampa, creazione di inchieste sul rilascio dei visti, etc.) e (3) la pressione politica al fine di giungere a cambiare alcune leggi e una modificazione delle pratiche (es.: nel corso della campagna elettorale 2012, insieme con l'ARDHIS ${ }^{11}$, il coordinamento nazionale ha messo a punto una piattaforma di rivendicazioni, comuni alle coppie etero ed omosessuali, da sottoporre ai futuri candidati premier ottenendo risposte o incontri da quattro candidati; una nota al ministero dell'interno è attualmente in redazione) (cfr. La Cimade e Les Amoureux au Ban, 2008).

L'associazione agisce, dunque, a livello nazionale, locale mediante la presenza di una struttura di collettivi, in rete grazie al Forum accessibile dal sito internet dell'associazione ${ }^{12}$ che permette a membri di scambiarsi informazioni sulle novità legislative e consigli operativi. La lobbying mediatico-politica resta dunque l'obiettivo ultimo dell'associazione il cui operato va al di là della semplice risoluzione di singoli casi, ma spera di permettere il cambiamento delle pratiche legislative in atto.

\subsection{L'esperienza spagnola: ASFAMIX}

Il movimento associativo di famiglie miste, o bi-nazionali, è nato nel 2005 e denominato inizialmente ASFADIS (Asociación de Familias Discriminadas), per lottare contro le discriminazioni, a carico dei minori nati da padre spagnolo e da madre straniera, causate dalla legge $35 / 2007$, che aiutava, con un assegno di 2.500 euro, le madri per la nascita di un figlio o per un'adozione. A questo assegno non potevano però accedere quelle famiglie dove il padre era spagnolo e la madre straniera se questa non risiedeva da almeno 2 anni in Spagna. Successivamente, nel 2009, si costituisce come associazione nazionale con il nome di ASFAMIX (Asociación de Familias Mixtas de España).

I principali obiettivi di ASFAMIX sono legati alla prevenzione o alla denuncia delle discriminazioni o dei disservizi incontrati dalle famiglie miste, offre sostegno giuridico e psicologico, promuove la creazione, nelle varie regioni spagnole, di altre associazioni locali che abbiano gli stessi obiet-

${ }^{11}$ www.ardhis.org Association pour la Reconnaissance des Droits des personnes Homosexuelles et Transsexuelles à l'immigration et au séjour.

$12 \mathrm{http}: / /$ www.amoureuxauban.net/ 
tivi di ASFAMIX, e più in generale, ha come obiettivo il benessere delle famiglie miste.

ASFAMIX in quattro anni di esperienza associativa, ha individuato, in Spagna, quattro grandi "focolai" di problemi: (1) Norme nazionali, che sono discriminatorie, come la citata legge $35 / 2007$; alcune restrizioni in materia di accesso a case popolari; la negazione degli sconti sui biglietti aerei per gli stranieri, anche se membri di famiglie miste, che risiedono fuori dalla penisola ma residenti nel territorio spagnolo (isole Canarie, Baleari, Ceuta e Melilla), ecc. (2) Legislazione internazionale. Essendo le legislazioni divergenti in materia di famiglia, ci possono essere gravi problemi per le coppie con figli minori a carico o in processo di separazione o divorzio. In questi casi, anche in presenza di una sentenza di custodia emessa da un paese, può sussistere la possibilità che il paese dell'altro coniuge non riconosca la sentenza, con gravi pregiudizi per i figli, arrivando, in casi estremi alla sottrazione di minori; (3) Relazione con le amministrazioni. In questa categoria si annoverano tutte le difficoltà inerenti al riconoscimento di documenti (titoli di studio, patenti, ecc.). In alcuni casi, la scarsa formazione o aggiornamento professionale dei funzionari può aggravare la situazione e causare disservizi o, in casi estremi, negare l'erogazione stessa del servizio alle famiglie miste. Inoltre, la scarsa formazione degli operatori dei servizi sociali ed educativi, spesso non è all'altezza per comprendere la peculiarità delle famiglie interculturali e quindi per offrire dei servizi su misura (Moscato, 2008). (4) Discriminazione in ambito privato a causa delle scarse conoscenze legislative. Per esempio, si sono manifestati casi di discriminazione, soprattutto legati al NIE (Numero de Identificación de Extranjeros), che vale come un documento di riconoscimento. In realtà, l'utilizzo di questo documento può creare gravi pregiudizi, come l'impossibilità di accedere alle offerte di lavoro, la negazione dell'imbarco su un volo nazionale o la negazione di un contratto telefonico.

Tra glì obiettivi a medio termine di ASFAMIX c'è la creazione di una rete multidisciplinare con anime dall'ambito accademico e istituzionale per promuovere progetti pilota in ambito psicosociale e comunitario, con il fine ultimo di migliorare i servizi per le famiglie. Infine, un altro progetto in cantiere è la creazione di una rete europea che abbia il peso sufficiente per correggere le tensioni legislativo-politiche che incidono sulle vite delle famiglie miste. 

schaften

L'associazione delle famiglie e delle coppie bi-nazionali ${ }^{13}$ (iaf e.V.) è stata fondata nel 1972 e garantisce la rappresentanza degli interessi delle famiglie miste a livello nazionale. L'obiettivo principale dell'associazione è che le persone e le famiglie siano socialmente e legalmente parificate, senza discriminazione sulla base della loro origine geografica, culturale, etnica o nazionale. Infatti, l'associazione tedesca lavora per facilitare e delineare prospettive chiare per una convivenza pacifica tra persone di culture differenti. Una delle rivendicazioni concerne la richiesta di una maggiore considerazione del pluri-linguismo nel processo educativo e formativo dei figli.

La sede centrale è a Francoforte, ma esistono altri venticinque gruppi sul territorio tedesco, nove dei quali hanno almeno un operatore assunto, mentre gli altri lavorano grazie al contributo di volontari. Tuttavia tutti offrono consulenze alle coppie bi-nazionali. La tipologia della consulenza varia a seconda dei bisogni locali (es.: predominanza di famiglie monoparentali, padri divorziati) e/o delle richieste delle autorità comunali che, talvolta, supportano e finanziano il lavoro dei gruppi. In particolare, iaf e. $V$. offre consulenze per uomini a seguito di separazione o divorzio, consulenze terapeutiche o psicologiche con psicoterapeuti professionisti, consulenze legali con avvocati e giuristi specializzati, consulenze e offerte di comunicazione per famiglie monoparentali, uno sportello specifico è dedicato a migranti adulti che vanno in Germania per la prima volta a Francoforte. Inoltre, il gruppo di Monaco di Baviera lavora come un consultorio istituzionalmente finanziato per consulenze matrimoniali, familiari e di vita. Infine, da anni il gruppo di Berlino lavora sul tema dell'apertura interculturale dei comuni o degli enti pubblici e sulle consulenze multiculturali e interculturali.

\section{Conclusioni}

Le prospettive operative aperte dalle esperienze associative mostrano la necessità di una maggiore comprensione del fenomeno, che rappresenta una realtà crescente in Europa, da parte delle autorità locali e nazionali e l'urgenza di fornire risposte adeguate. Benché l'Unione Europea non abbia competenza per legiferare, essa può contribuire al benessere di queste famiglie promuovendo raccomandazioni e linee di indirizzo politico dirette

${ }^{13}$ Per ulteriori informazioni consultare il sito dell'Associazione alla pagina http://www. verband-binationaler.de/ 
agli Stati Membri. Concretamente, può supportare queste iniziative creando gruppi di lavoro ad hoc e peer reviews a livello europeo, dando così impulso a una discussione e a una condivisione di buone pratiche. Infine, l'Unione Europea potrebbe includere la tematica tra le priorità di finanziamento, sia nei programmi di ricerca, sia nei fondi strutturali, in particolare nel Fondo Sociale Europeo, per programmi di formazione e aggiornamento per funzionari e professionisti. Tuttavia, il nodo rimane politico. Soltanto con un cambiamento di prospettiva e con delle azioni congiunte sarà possibile arrivare a un cambiamento reale. Per questo è importante che i cittadini e le diverse associazioni non si fermino ma anzi continuino il cammino intrapreso, facendo sentire la loro voce e affiancando azioni di lobbying a azioni di supporto concreto.

\section{Riferimenti bibliografici}

Collet B. (2012). Mixed Couple in France. Statistics Facts, Definitions, and Social Reality, Papers Revista de sociologia, 97, 1, 91-77.

Eurostat (2012). Statistics in focus 29/2012.

Ferran N. (2009). La politique d'immigration contre les couples mixtes. In: Le Cour Gradnmaison O. Douce France. Rafles, rétention, expulsions, Paris, Seuil, coll. «Essais», pp. 151-172.

Gisti, La Cimade et Les Amoureux au Ban Public (2009). Le mariage des étrangers. Paris: Les cahiers juridiques du Gisti.

La Cimade (2010). Visa refusé. Enquête sur les pratiques de consulats de France en matière de délivrance des visas. Rapport d'observation.

La Cimade e Les Amoureux au Ban (2008). Peu de meilleur et trop de pire. Soupçonnés, humiliés, réprimés des couples mixtes témoignent. Rapport d'observation.

Moscato G. (2008). Parejas mixtas e inmigración: apoyo social, gestión de diferencias culturales, y satisfacción marital. Tesi pre-dottorale no publicada, Universidad de Málaga.

Moscato G. (2012). Familias interculturales en España: análisis de la satisfacción vital. Portularia, 12, 35-43. DOI: 10.5218/PRTS.2012.0004

Odasso L. (2013). La mixité conjugale: une expérience de migration. Approche comparée des effets de la stigmatisation sur les natifs et leurs partenaires «arabes» en Vénétie et en Alsace. Tesi di dottorato, Università Ca' Foscari, Venezia e Université de Strasbourg. 